\title{
Sequential improvement in paediatric medulloblastoma outcomes in a low-and-middle-income country setting over three decades
}

\begin{tabular}{|c|c|}
\hline \multicolumn{2}{|c|}{$\begin{array}{l}\text { Authors: } \\
\text { Johann Riedemann } n^{1,2} \\
\text { Anthony Figaji } \\
\text { Alan Davidson }^{4} \\
\text { Clare Stannard }^{4} \\
\text { Komala Pillay }^{5,6} \\
\text { Tracy Kilborn }^{7} \\
\text { Jeannette Parkes } \\
{ }^{1,2}\end{array}$} \\
\hline \multicolumn{2}{|c|}{$\begin{array}{l}\text { Affiliations: } \\
{ }^{1} \text { Department of Radiation } \\
\text { Oncology, Faculty of } \\
\text { Radiation Medicine, } \\
\text { University of Cape Town, } \\
\text { Cape Town, South Africa }\end{array}$} \\
\hline \multicolumn{2}{|c|}{$\begin{array}{l}{ }^{2} \text { Department of Radiation } \\
\text { Oncology, Faculty of } \\
\text { Radiation Medicine, Groote } \\
\text { Schuur Hospital, Cape Town, } \\
\text { South Africa }\end{array}$} \\
\hline \multicolumn{2}{|c|}{$\begin{array}{l}{ }^{3} \text { Division of Neurosurgery } \\
\text { and Neuroscience Institute, } \\
\text { Faculty of Neurosurgery, Red } \\
\text { Cross War Memorial } \\
\text { Children's Hospital, University } \\
\text { of Cape Town, Cape Town, } \\
\text { South Africa }\end{array}$} \\
\hline \multicolumn{2}{|c|}{$\begin{array}{l}{ }^{4} \text { Department of Haematology } \\
\text { Oncology Service, Faculty of } \\
\text { Paediatrics and Child Health, } \\
\text { Red Cross War Memorial } \\
\text { Children's Hospital, Cape } \\
\text { Town, South Africa }\end{array}$} \\
\hline \multicolumn{2}{|c|}{$\begin{array}{l}{ }^{5} \text { Division of Anatomical } \\
\text { Pathology, Anatomical } \\
\text { Pathology Service, National } \\
\text { Health Laboratory Services, } \\
\text { South Africa }\end{array}$} \\
\hline \multicolumn{2}{|c|}{$\begin{array}{l}{ }^{6} \text { Faculty of Anatomical } \\
\text { Pathology, Red Cross War } \\
\text { Memorial Children's Hospital, } \\
\text { University of Cape Town, } \\
\text { Cape Town, South Africa }\end{array}$} \\
\hline \multicolumn{2}{|l|}{ Read online: } \\
\hline 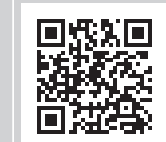 & $\begin{array}{l}\text { Scan this QR } \\
\text { code with your } \\
\text { smart phone or } \\
\text { mobile device } \\
\text { to read online. }\end{array}$ \\
\hline
\end{tabular}

Background: Medulloblastoma (MB) is the commonest malignant brain tumour of childhood. Accurate clinical data on paediatric MB in the low-and-middle-income countries (LMIC) setting are lacking. Sequential improvements in outcomes seen in high-income countries are yet to be reflected in LMICs.

Aim: The aim of this study was quantification of paediatric MB outcomes in the LMIC setting over three decades of advances in multidisciplinary intervention.

Setting: Cape Town, South Africa.

Methods: This was a retrospective study of 136 children with MB diagnosed between 1985 and 2015. The modified Chang criteria were used for risk stratification. The primary objective of this study was overall survival (OS), quantified by analysis of epidemiological, clinical and pathological data.

Results: OS improved significantly during the most recent decade (2005-2015) when compared with the preceding two decades (1985-1995 and 1995-2005). Despite reduced-dose craniospinal irradiation (CSI) for standard risk cases, OS was significantly greater than during the preceding two decades. High-risk disease was identified in $71.4 \%$ of cases and was associated with significantly inferior OS compared with standard-risk cases. Improved OS was positively correlated with the therapeutic era, three-dimensional (3D) conformal radiotherapy technique, older age at diagnosis, classic and desmoplastic histology, extent of resection and absence of leptomeningeal spread on imaging.

Conclusion: Advances in multidisciplinary management of MB in our combined service are associated with improved survival. Access to improved imaging modalities, advances in surgical techniques, increased number of patients receiving risk-adapted combination chemotherapy or radiotherapy, as well as CSI using a linear accelerator with 3D planning, are considered as contributing factors.

Keywords: Paediatric brain tumours; medulloblastoma; radiotherapy; 3D conformal radiotherapy; craniospinal irradiation; chemotherapy; paediatric neurosurgery;leptomeningeal spread; cerebrospinal fluid; paediatric oncology; low-and-middle-income countries; Paediatric Oncology in Developing Countries; overall survival.

\section{Introduction}

Brain and central nervous tumours are rare in children but result in mortality and morbidity that is disproportionate to incidence rates. They constitute the leading cause of paediatric cancerassociated deaths worldwide, despite lower incidence rates in comparison with haematological malignancies. ${ }^{1}$

A recent report analysing paediatric cancer survival rates in two South African tertiary hospitals revealed that brain tumours represent $19.5 \%$ of all childhood cancers diagnosed, which is second only to leukaemia constituting $25 \%$ of cases. According to international reports, those with brain

\footnotetext{
${ }^{7}$ Department of Radiology, Red Cross War Memorial Children's Hospital, University of Cape Town, Cape Town, South Africa Corresponding author: Johann Riedemann, rieders2013@gmail.com

Dates: Received: 28 Feb. 2021 | Accepted: 08 Mar. 2021 | Published: 26 May 2021

How to cite this article: Riedemann J, Figaji A, Davidson A, et al. Sequential improvement in paediatric medulloblastoma outcomes in a low-and-middle-income country setting over three decades. S. Afr. j. oncol. 2021;5(0), a174. https://doi.org/10.4102/sajo.v5i0.174

Copyright: @ 2021. The Authors. Licensee: AOSIS. This work is licensed under the Creative Commons Attribution License.
} 
tumours exhibited the lowest survival rate with a median survival time of 18 months compared with 47 months for leukaemia cases. ${ }^{1,2}$

Medulloblastoma (MB) is the most common malignant paediatric brain tumour, comprising a group of histologically and molecularly diverse posterior fossa tumours, pathologically described as undifferentiated, small round blue cells with mild-to-moderate nuclear pleomorphism and high mitotic counts. These tumours are of embryonic origin and are believed to have originated from progenitor cell lineages present during early brain development. The following four histological variants exist: classic, desmoplastic or nodular, MB with extensive nodularity (MBEN) and largecell or anaplastic (LCA) histology. In addition, four molecular subgroups, including wingless (WNT), sonic hedgehog (SHH), Group 3 and Group 4, have been identified. 3,4,5,6,7 Molecular subgrouping may influence treatment decisionmaking and is a strong predictor of prognostic outcomes. ${ }^{5,8,9}$

Approximately $20 \%$ of paediatric brain tumours in highincome countries (HICs) are MB. Data from low-and-middleincome countries (LMICs) exhibit a substantial variation in incidence rates ranging from $6 \%$ to $50 \%{ }^{10,11,12,13,14}$ The exact epidemiological, clinicopathological characteristics and survival outcomes of MB within South Africa's markedly heterogeneous population are unknown. The risk stratification of children with MB using the modified Chang system allows for identification of standard- and high-risk groups. ${ }^{15}$ Standard risk is defined as follows: age $\geq 3$ years at presentation, $<1.5 \mathrm{~cm}^{2}$ residual tumour after resection, cerebrospinal fluid (CSF) negative for tumour cells, absence of leptomeningeal spread on computed tomography (CT) or magnetic resonance imaging (MRI) and classic or desmoplastic histology. High-risk disease is defined when any of the following are present: age $<3$ years, residual tumour of $>1.5 \mathrm{~cm}^{2}$ after surgery, CSF positive for tumour cells, leptomeningeal spread on CT / MRI or LCA histology. ${ }^{15,16}$ Over the last decade, advances in the multidisciplinary management of patients with MB in HICs have resulted in 5 -year overall survival (OS) rates of $80 \%-85 \%$ for standardrisk and $65 \%-70 \%$ for high-risk disease. ${ }^{16,17}$ This improvement is not apparent in LMICs and may be attributed to common constraints, such as delayed or inaccurate diagnoses with advanced disease at presentation, high rates of hospital acquired infections following neurosurgical intervention and co-morbid illnesses, such as HIV, TB, parasitic disease and malnutrition. Limited access to radiotherapy (RT) services with significant delays from referral to point of treatment, as well as the ever-expanding limitations within socio-economic support structures, further hampers optimal care. . $11,18,19^{2}$

In order to quantify treatment outcomes associated with $\mathrm{MB}$ in the context of a LMIC, we undertook a 30-year review of all children (aged 0-15), who were diagnosed with MB between years 1985 and 2015. They underwent multidisciplinary management at the Red Cross War Memorial and Groote Schuur Hospital complex. Demographic and clinicopathological characteristics were recorded and related to OS.

\section{Methods Cohort and clinical data}

A 30-year retrospective, comparative assessment of 136 patients with MB aged 0-15 years, who were treated at Red Cross War Memorial and Groote Schuur Hospital complex between 1985 and 2015, was performed. Information obtained from records of oncology, neurosurgery and RT patient was cross-referenced to maximise data capture and ensure accuracy. Information retrieved included age, sex, date of birth, date of diagnosis, presenting clinical features, histology, radiology reports, extent of resection, CSF analysis, chemotherapy and RT schedules. Cerebrospinal fluid obtained were either intraoperative cisternal puncture or day 14 post-operative lumbar puncture samples. Because of the duration of this study and associated constraints of missing patient records, we were unable to accurately ascertain the original source of CSF fluid. These limitations also resulted in numbers for the respective subgroups not equating to the total population number of 136. Missing data are, therefore, included in the data tables. Laboratory results were obtained from printed copies in the patient record or from accessing the South African National Health Laboratory Service DisaLab (up to July 2013) or TrakCare (August 2013 December 2015) platforms. Imaging results were obtained from printed radiology reports, clinical notes and accessing iSite Enterprise Radiology PACS. Prior to 1992, only CT scans were available. Between the years 1992 and 2001, patients received CT scans at Red Cross War Memorial Hospital and MRI scans at the local Medical Research Council facility. Between the years 2001 and 2009, MRI scans were performed at Groote Schuur Hospital. An MRI scanner was introduced at the Red Cross War Memorial Hospital in 2009. Prior to the year 2012, contrasted CT was used to evaluate for leptomeningeal spread during the initial diagnostic assessment. During the later part of this study, MRI was predominantly used. Over time, an immediate post-operative MRI was used to determine surgical outcomes. Using the modified Chang criteria, cases were stratified into standard and high-risk groups. ${ }^{15}$ The combined data were further cross-referenced with the South African Children's Cancer Study Group (SACCSG) registry.

\section{Radiotherapy}

During the first two decades (1985-2005), two-dimensional (2D) planning in combination with $\mathrm{a}^{60}$ Co gamma ray unit was used. From 2005 to 2015, all cases were 3D planned with $6 \mathrm{MV}$ photon energy and treated with a linear accelerator (LINAC). From 2007, standard-risk MB was treated with reduced-dose craniospinal irradiation (CSI) consisting of 23.4 gray (Gy) to the craniospinal axis in 13 fractions together with a tumour bed boost of 30.6 Gy - 32.4 Gy in 17-18 fractions. This is in accordance with the Pediatric Oncology in Developing Countries (PODC) Committee of the International Society of Pediatric Oncology (SIOP) adapted treatment recommendations. ${ }^{11}$ High-risk disease was treated with a full-dose CSI of $36 \mathrm{~Gy}$ in 20 fractions, with a boost dose of 18.0 Gy - 19.8 Gy in 10-11 fractions to the tumour bed. 
Selected cases harbouring a significant, gross residual disease occasionally necessitated an additional boost depending on the dose to surrounding organs at risk.

\section{Extent of resection}

Prior to the year 2011, contrast-enhanced CT was used for post-operative imaging (usually the day after surgery) and was subsequently replaced by immediate post-operative MRI ( $<48 \mathrm{~h}$ post-surgery) imaging during the later part of this study. Gross total resection (GTR) was defined as total resection of the primary tumour without residual lesions as reported by the neurosurgeon and post-surgical imaging when available. Near total resection (NTR) was defined as $<1.5 \mathrm{~cm}^{2}$ residual tumour, whereas sub-total resection (STR) represented an incomplete resection with $>1.5 \mathrm{~cm}^{2}$ residual tumour.

\section{Chemotherapy}

Data captured reflect administration of chemotherapy at any point of the patient's journey. During the early phases of this study, chemotherapy was not routinely prescribed, except for high-risk cases, which is most commonly reported in young children under the age of 3-4 years, in whom the expected long-term sequelae of craniospinal RT necessitated delay of RT and the use of alternative treatment modalities. From 1985 to 1995, all high-risk patients received chemotherapy, either because the start of CSI was delayed, or adjuvantly, or both, but this was given at Groote Schuur Hospital. In the second cohort, high-risk patients received adjuvant vincristine, etoposide and cyclophosphamide (VEC) at Red Cross War Memorial Hospital, and after the year 2005, all high-risk patients and those with standard risk received reduced-dose CSI together with concurrent and adjuvant chemotherapy. After the year 2015, concurrent weekly vincristine was omitted.

\section{Tissue preparation and immunohistochemistry}

Tumour specimens were processed by anatomical pathology as per the standard hospital protocol. Histological phenotypic subtyping was performed using standard procedures as previously described..$^{20}$

\section{Statistical analysis}

Statistical analysis was performed using the GraphPad Prism software. The Kaplan-Meier methodology was applied to quantify survival outcomes. Log-rank tests were used to determine differences between groups. ${ }^{21}$ Overall survival was calculated as the time from the date of diagnosis to death from any cause or last contact. Chi-square $\left(\chi^{2}\right)$ tests were performed to measure differences between derived proportions. In order to evaluate the potential influence of each risk constituent on outcome, OS for age, histological subtype, CSF status, leptomeningeal spread and extent of resection was calculated. Univariate Cox regression analysis of OS determinants was performed to quantify confidence intervals and hazard ratios that are graphically depicted on a Forest plot.

\section{Ethical considerations}

Ethics approval was obtained from the Human Research Ethics Committee, University of Cape Town (Reference 777/2018 linked to sub-study 149/2014). Approval renewed 28.8.2020.

\section{Results}

\section{Study population and demographics}

About 136 patients who had undergone multidisciplinary management for MB between 1985 and 2015 were identified. Demographic and clinical characteristics of the MB cohort are summarised in Table 1 . The male-to-female ratio was 0.9 , and the median ages at diagnosis were 2.1 and 7.3 years for the 0-3 years group, and 3-15 years groups, respectively, with a combined median age of 5.7 years. The standard-risk cases were $34 / 119(28.6 \%)$ of the entire cohort and 85/119 (71.4\%) were high-risk cases, whereas 17 cases could not be accurately staged. Histologically, the evaluable sub-cohort consisted of 93/128 (72.7\%) classic histology, 22/128 (17.2\%) desmoplastic or nodular or MBEN and 13/128 (10.1\%) LCA variants. The results of CSF analysed revealed that 22/93 (23.7\%) cases were positive for malignant cells. Because of the constraints associated with the duration of this study, we were unable to determine the origin of the CSF samples, and results reflect either intraoperative cisternal puncture or day 14 post-operative lumbar puncture samples. Baseline diagnostic imaging of the spinal axes (either CT or MRI depending on era) at presentation exhibited features consistent with leptomeningeal disease or drop metastases in 18 out of 90 (20.0\%) evaluable cases.

\section{Survival analysis}

The 5-year and 10-year OS rates for the entire cohort was $60.5 \%$ and $54.6 \%$, respectively (Figure 1). Survival was improved for the most recent decade 2005-2015, in comparison with the preceding two decades (Log-rank test $p=0.0423 ; n=136$ ). The 5-year OS (OS5) rates for the first,

TABLE 1: Demographic and clinical characteristics of the medulloblastoma study cohort.

\begin{tabular}{|c|c|c|c|c|c|c|}
\hline \multirow[t]{2}{*}{ Variable } & \multicolumn{2}{|c|}{ Age $<3$} & \multicolumn{2}{|c|}{ Age 3-15 } & \multicolumn{2}{|c|}{ All patients } \\
\hline & $n$ & $\%$ & $n$ & $\%$ & $n$ & $\%$ \\
\hline Number $(n=136)$ & $36 / 136$ & 26.5 & $100 / 136$ & 73.5 & 136 & - \\
\hline Incidence (per annum) & 1.21 & - & 3.31 & - & 4.52 & - \\
\hline Gender ratio (Male:Female) & 0.90 & - & 0.93 & - & 0.91 & - \\
\hline $\begin{array}{l}\text { Median age at diagnosis } \\
\text { (years) }\end{array}$ & 2.1 & - & 7.3 & - & 5.7 & - \\
\hline \multicolumn{7}{|l|}{ Histology $(\%) n=128$} \\
\hline Classic & $24 / 35$ & 68.6 & $69 / 93$ & 74.2 & $93 / 198$ & 72.7 \\
\hline Desmoplastic / Nodular & $7 / 35$ & 20.0 & $15 / 93$ & 16.1 & $22 / 128$ & 17.2 \\
\hline Large Cell Anaplastic & $4 / 35$ & 11.4 & $9 / 93$ & 9.7 & $13 / 128$ & 10.1 \\
\hline \multicolumn{7}{|l|}{ CSF metastases $(\%) n=93$} \\
\hline Positive & $5 / 21$ & 23.8 & $17 / 72$ & 23.6 & $22 / 93$ & 23.7 \\
\hline Negative & $16 / 21$ & 76.2 & $55 / 72$ & 74.4 & $71 / 93$ & 76.3 \\
\hline \multicolumn{7}{|c|}{ Leptomeningeal spread $(\%) n=90$} \\
\hline Positive & $4 / 21$ & 19.0 & $14 / 69$ & 20.3 & $18 / 90$ & 20.0 \\
\hline Negative & $17 / 21$ & 81.0 & $55 / 69$ & 79.7 & $72 / 90$ & 80.0 \\
\hline \multicolumn{7}{|c|}{ Modified Chang Risk Stratification $(\%) n=119$} \\
\hline Standard & $0 / 36$ & 0.0 & $34 / 83$ & 41.0 & $34 / 119$ & 28.6 \\
\hline High & $36 / 36$ & 100.0 & $49 / 83$ & 59.0 & $85 / 119$ & 71.4 \\
\hline
\end{tabular}

CSF, cerebrospinal fluid. 


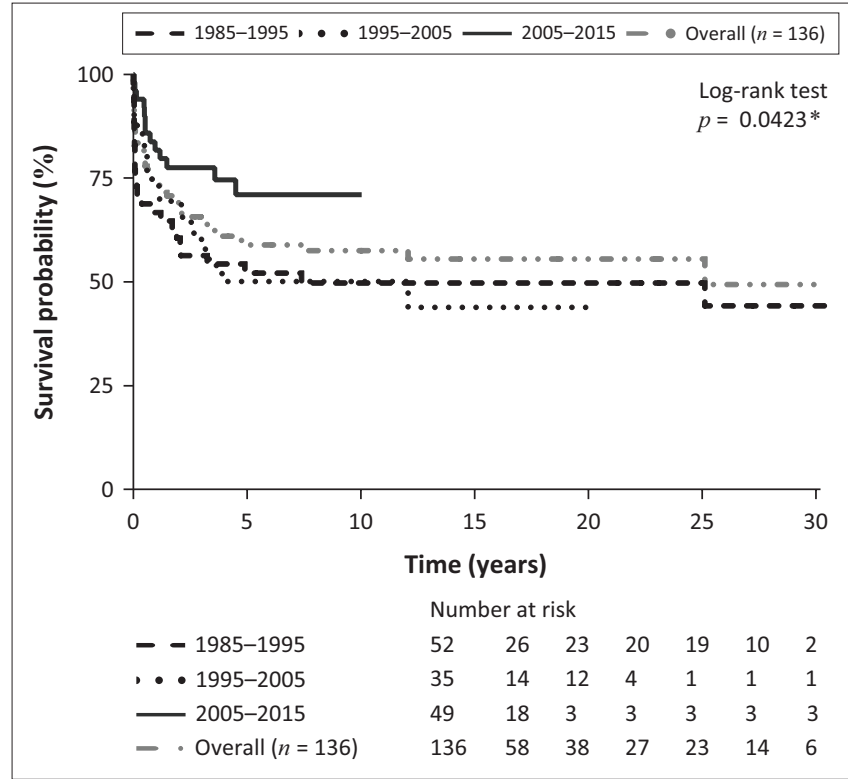

*, equal or less that 0.05 .

FIGURE 1: Kaplan-Meier plots illustrating overall survival during three therapeutic eras for the entire cohort $(n=136)$.

second and third decades were 51.5\% (95\% confidence interval [CI] 39.6-66.6; $n=52), 49.3 \%(95 \%$ CI 33.6-64.8; $n=35)$ and $76.2 \%(95 \%$ CI $68.7-84.7 ; n=49)$, respectively. The 10-year OS (OS10) rates for the first, second and third decades were $49.5 \%$ (95\% CI $23.6-58.5 ; n=52), 49.1 \%$ (95\% CI $33.4-$ $63.4 ; n=35)$ and $73.2 \%(95 \%$ CI $67.1-89.6 ; n=49)$, respectively. The OS5 rate during the decade 2005-2015 was significantly higher compared with the decades 1985-1995 ( $p=0.0186$ ) and 1995-2005 ( $p=0.0319)$ when individually compared. No significant difference in OS was observed for periods 1985-1995 versus 1995-2005 ( $p=0.5650)$.

An unequal risk distribution amongst therapeutic groups can influence survival assessment and interpretation. Table 2 summarises the prevalence of measurable variables within each group. The decade 2005-2015 was characterised by a significantly greater number of cases with the absence of leptomeningeal spread $(p=0.001)$, standard-risk disease $(p=0.0048)$ and GTR $(p=0.0310)$, whereas STR occurred less frequently $(p=0.003)$.

Across the entire cohort, standard-risk MB was associated with significantly superior OS compared with high-risk disease (Figure 2a). The OS5 and OS10 rates of standard-risk patients were $78.7 \%$ (95\% CI $64.7-89.7)$ and $70.4 \%$ (95\% CI 60.5-88.6), respectively, compared with 51.2\% (95\% CI 39.6-58.3) and $45.7 \%$ (95\% CI 33.6-57.2) for high-risk disease (OS5; $p=0.0026 ; n=117)$. Incompletely staged cases constituted 19/136 (13.9\%).

OS was significantly different between the age groups (Figure 2b). OS5 and OS10 rates for age group 0-3 years were $32.5 \%$ (95\% CI 18.2-51.9) for both decades, compared with the age group 3-15 years, with OS5 and OS10 rates of 67.1\% (95\% CI 54.5-73.8) and 59.8\% (95\% CI 47.3-70.1), respectively (OS5; $p=0.0008 ; n=136)$.
TABLE 2: Risk factor distribution between therapeutic eras.

\begin{tabular}{|c|c|c|c|c|c|c|c|}
\hline \multirow{2}{*}{$\begin{array}{l}\text { Total number per } \\
\text { decade } \\
n=136\end{array}$} & \multicolumn{2}{|c|}{$\begin{array}{c}1985-1995 \\
n=52\end{array}$} & \multicolumn{2}{|c|}{$\begin{array}{c}1995-2005 \\
n=35\end{array}$} & \multicolumn{2}{|c|}{$\begin{array}{c}2005-2015 \\
n=49\end{array}$} & \multirow[t]{2}{*}{$p$-value } \\
\hline & $n$ & $\%$ & $n$ & $\%$ & $n$ & $\%$ & \\
\hline \multicolumn{8}{|c|}{ Chang risk stratification } \\
\hline Standard & 7 & 13.5 & 7 & 20.0 & 20 & 40.8 & $0.0048 * *$ \\
\hline High & 32 & 61.5 & 22 & 62.9 & 29 & 59.2 & 0.9394 \\
\hline Incomplete & 13 & 25.0 & 6 & 17.1 & 0 & 0.00 & N/A \\
\hline \multicolumn{8}{|l|}{ Age (years) } \\
\hline $0-3$ & 15 & 28.8 & 11 & 31.4 & 10 & 20.4 & 0.8640 \\
\hline $3-15$ & 37 & 71.2 & 24 & 68.6 & 39 & 79.6 & 0.8911 \\
\hline \multicolumn{8}{|l|}{ Histology } \\
\hline Classic & 37 & 71.2 & 26 & 74.3 & 30 & 61.2 & 0.3848 \\
\hline Desmoplastic & 10 & 19.2 & 5 & 14.3 & 7 & 14.3 & 0.7486 \\
\hline $\begin{array}{l}\text { Large-cell anaplastic } \\
\text { variant }\end{array}$ & 0 & 0.0 & 1 & 2.9 & 12 & 24.5 & N/A \\
\hline Unknown & 5 & 9.6 & 3 & 8.5 & 0 & 0.0 & N/A \\
\hline \multicolumn{8}{|l|}{ Extent of resection } \\
\hline GTR & 16 & 30.8 & 10 & 28.6 & 23 & 46.0 & $0.0310 *$ \\
\hline NTR & 11 & 21.2 & 8 & 22.9 & 17 & 41.0 & 0.3507 \\
\hline STR/Biopsy & 20 & 38.5 & 15 & 42.9 & 5 & 12.0 & $0.003 * *$ \\
\hline Unknown & 5 & 9.5 & 2 & 5.7 & 4 & 8.2 & N/A \\
\hline \multicolumn{8}{|l|}{ CSF metastases } \\
\hline Positive & 2 & 3.8 & 4 & 11.4 & 16 & 32.71 & N/A \\
\hline Negative & 21 & 40.4 & 18 & 51.4 & 32 & 65.3 & 0.6418 \\
\hline Unknown & 29 & 55.8 & 13 & 37.2 & 1 & 2.0 & N/A \\
\hline \multicolumn{8}{|c|}{ Leptomeningeal spread } \\
\hline Positive & 9 & 17.3 & 4 & 11.4 & 5 & 10.2 & 0.5373 \\
\hline Negative & 14 & 26.9 & 17 & 48.6 & 41 & 83.7 & $0.001 * *$ \\
\hline Unknown & 29 & 55.8 & 14 & 40.0 & 3 & 6.1 & NA \\
\hline
\end{tabular}

CSF, cerebrospinal fluid; N/A, not applicable due to small sample size; GTR, Gross total resection; NTR, Near Total Resection; STR, sub-total resection.

*, equal or less than 0.05 ; **, equal or less than $0.01 ; * * *$, equal or less than 0.001 .

LCA histology was associated with significantly inferior OS compared with classic and desmoplastic variants $(p=0.0347$; $n=128$; Figure 2c). Both OS5 and OS10 rates were 39.5\% (95\% CI 20.8-54.9) for cases with LCA histology. In comparison, classic histology exhibited OS5 and OS10 rates of 66.1\% (95\% CI 54.5-74.7) and 60.5\% (95\% CI 51.2-73.1), respectively, whereas desmoplastic histology was associated with OS5 and OS10 rates of 58.9\% (95\% CI 38.5-77.1) and 52.8\% (95\% CI 30.3-70.6; $p=0.298)$.

No significant differences in OS were observed for patients with or without malignant cells in CSF samples ( $p=0.8106$; $n=93$; Figure 2d); however, the presence of leptomeningeal spread on preoperative diagnostic imaging was associated with reduced survival (Figure 2e). The OS5 and OS10 rates in the absence of leptomeningeal spread were $69.7 \%$ (95\% CI 57.6-78.7) and $64.8 \%$ (95\% CI 57.3-78.7), respectively. Leptomeningeal spread was associated with OS5 and OS10 rates of $49.6 \%$ (95\% CI 33.7-55.4) and 33.9\% (95\% CI $17.3-52.2)$, respectively ( $p=0.0482 ; n=90)$. We were unable to accurately document $46 / 136$ (33.8\%) cases for leptomeningeal involvement.

Maximal safe surgical resection was performed by specialised paediatric neurosurgeons throughout the study period. The OS5 and OS10 rates for GTR cases were $69.6 \%$ and $66.5 \%$, respectively, compared with $55.5 \%$ and $49.4 \%$ for NTR cases $(p=0.2810)$. The OS5 and OS10 rates for STR cases were $48.4 \%$ and $43.8 \%$, respectively, and were 


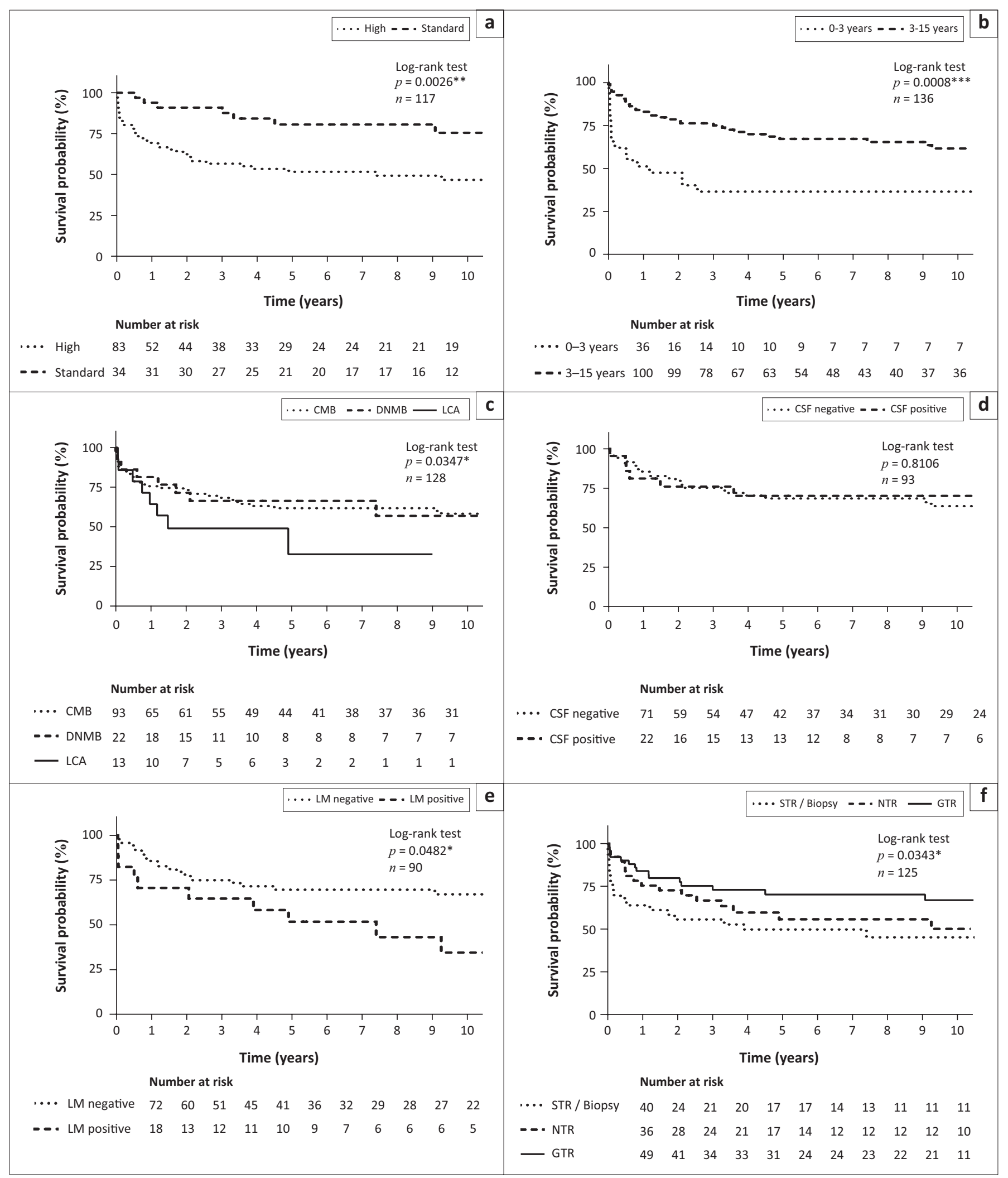

CMB, classic medulloblastoma; DNMB, desmoplastic nodular medulloblastoma; LCA, large cell anaplastic, LM, leptomeningeal; GTR, gross total resection; NTR, near total resection; STR, subtotal resection; CSF, cerebrospinal fluid.

*, equal or less than $0.05 ; * *$, equal or less than $0.01 ; * * *$, equal or less than 0.001 .

FIGURE 2: Overall survival in context of risk stratification including combined risk (a), age at diagnosis (b), histological subtype (c), cerebrospinal fluid (CSF) results (d), leptomeningeal involvement (e) and extent of resection (f). The evaluable sub-cohort sizes are indicated $(n)$.

significantly lower than those for GTR cases $(p=0.0343$; $n=125$; Figure 2f). Of the 136 cases, we were unable to ascertain the extent of resection in 11 cases.
Survival was not significantly different between male and female patients; however, a trend towards increased survival was observed for female patients (Supplemental Figure A). 
The OS5 and OS10 rates of female patients were 66.8\% (95\% CI 61.1-72.3) and 61.6\% (95\% CI 54.7\%-67.9\%), respectively, compared with male patients with OS5 and OS10 rates of $51.7 \%$ (95\% CI $44.1-59.2)$ and $48.4 \%$ (95\% CI $40.7-55.1$; $p=0.1070 ; n=136)$, respectively. Risk stratification and treatment modalities in relation to the male and female subcohorts were quantified (Supplemental Table A). Classic histology was observed in 55/67 (82.1\%) female patients compared with $38 / 61(62.3 \%)$ male counterparts ( $p=0.0168$; $n=128)$. Only $2 / 67(3 \%)$ female patients harboured LCA histology, significantly fewer than their male counterparts with $11 / 61(18.0 \%)(p=0.0068 ; n=128)$. No additional differences in relation to sex were observed.

Because of the study duration and resultant missing patient data, we could only with certainty evaluate RT outcomes in $100 / 136(73.5 \%)$ patients. Of these 44/100 (44\%), 21/100 (21\%) and 35/100 (35\%) were treated during the first, second and third decades, respectively. Fractionation and dose prescriptions are summarised in Table 3. Linac-based 3D conformal RT (3DCRT), employed during the decade of 2005-2015, was associated with OS5 and OS10 rates of 76.2\% and $73.2 \%$, respectively. The combined OS5 and OS10 rates during the preceding two decades, when 2D Cobalt-60-based RT was used, were $59.4 \%$ and $57.8 \%$, respectively ( $p=0.0286$; $n=100$; Figure 3).

Surgical outcomes were evaluable in 125/136 (91.9\%; $n=125)$ cases. The number of GTR achieved during the decade 2005-2015 was 23/45 (51\%), which is significantly higher compared with $16 / 47$ (34\%) and 10/33 (30\%) during the decades 1985-1995 and 1995-2005, respectively ( $p=0.0310)$. There were significantly fewer STRs observed during the decade 2005-2015 (5/45), compared with the preceding two decades $(20 / 47$ and $15 / 33 ; p=0.0016)$. A non-significant trend towards greater NTR was observed during the period 2005-2015 ( $p=0.1180$; Table 3).

During the first, second and third decades, 26/37 (70\%), $21 / 25(84 \%)$ and $45 / 49(92 \%)$ cases received chemotherapy $\left(\chi^{2} ; p=0.439 ; n=111\right)$. We were unable to accurately quantify this variable in $25 / 136$ (18.4\%) cases, all occurring during the first two decades. From 1995 to 2005, high-risk patients fit for chemotherapy received VEC. During the most recent decade, standard-risk cases were treated with VEC, and high-risk cases received vincristine, carboplatin, etoposide alternating with vincristine, cisplatin, etoposide and cyclophosphamide.

Contrasted CT was the imaging modality available during the decade 1985-1995. MRI imaging only became available towards the end of the first decade with full implementation during the middle and last decades. Early post-operative MRI to determine the extent of resection only became a standard practice in 2011 (Table 3).

Univariate Cox regression analysis (Figure 4) of our data suggest that therapeutic decade 2005-2015 ( $p=0.0315)$, Linac
TABLE 3: Constituents of multidisciplinary management $(N=136)$

\begin{tabular}{|c|c|c|c|c|}
\hline Treatment & 1985-1995 & 1995-2005 & 2005-2015 & $p$-value \\
\hline Radiotherapy $(N)$ & 52 & 35 & 49 & - \\
\hline Cobalt-60 (2D) & 44 & 21 & - & - \\
\hline Linac 6MV (3D) & - & - & 35 & - \\
\hline Unknown & 8 & 14 & 14 & - \\
\hline Craniospinal dose & $\begin{array}{c}31.5 \mathrm{~Gy}- \\
36.8 \mathrm{GY}\end{array}$ & $\begin{array}{c}33.5 \mathrm{~Gy}- \\
37.0 \mathrm{~Gy}\end{array}$ & - & - \\
\hline Standard risk & - & - & $23.4 \mathrm{~Gy}$ & - \\
\hline High risk & - & - & $36.0 \mathrm{~Gy}$ & - \\
\hline Boost to tumour bed & $21.0 \mathrm{~Gy}$ & $\begin{array}{c}21.0 \mathrm{GY} \text { - } \\
24.1 \mathrm{~Gy}\end{array}$ & - & - \\
\hline Standard risk & - & - & $\begin{array}{c}30.6 \mathrm{~Gy}- \\
32.4 \mathrm{~Gy}\end{array}$ & - \\
\hline High risk & - & - & $\begin{array}{c}18.0 \mathrm{~Gy}- \\
19.8 \mathrm{~Gy}\end{array}$ & - \\
\hline Fractions per week & $4-5$ & $4-5$ & $4-5$ & - \\
\hline Dose per fraction & $\begin{array}{c}1.2 \mathrm{~Gy}- \\
1.7 \mathrm{~Gy}\end{array}$ & $\begin{array}{c}1.3 \mathrm{~Gy}- \\
1.9 \mathrm{~Gy}\end{array}$ & $1.8 \mathrm{~Gy}$ & - \\
\hline Surgery $(N)$ & 52 & 35 & 49 & - \\
\hline GTR & - & - & - & $0.0310^{*}$ \\
\hline GTR: 1985-1995 $(n=47)$ & 16 & - & - & - \\
\hline GTR: 1985-1995 (\%) & 31 & - & - & - \\
\hline GTR: $1995-2005(n=33)$ & - & 10 & - & - \\
\hline GTR: 1995-2005 (\%) & - & 30 & - & - \\
\hline GTR: 2005-2015 $(n=45)$ & - & - & 23 & \\
\hline GTR: 2005-2015 (\%) & - & - & 51 & \\
\hline NTR: & & & & 0.118 \\
\hline NTR: $1985-1995(n=47)$ & 11 & - & - & - \\
\hline NTR: 1985-1995 (\%) & 23 & - & - & - \\
\hline NTR: 1995-2005 $(n=33)$ & - & 8 & - & - \\
\hline NTR: 1995-2005 (\%) & - & 24 & - & - \\
\hline NTR: 2005-2015 $(n=45)$ & - & - & 17 & - \\
\hline NTR: 2005-2015 (\%) & - & - & 38 & - \\
\hline STR/Biopsy $(N)$ & - & - & - & $0.0016 * *$ \\
\hline STR/Biopsy: 1985-1995 $(n=47)$ & 20 & - & - & - \\
\hline STR/Biopsy: 1985-1995 (\%) & 42 & - & - & - \\
\hline STR/Biopsy: 1995-2005 $(n=33)$ & - & 15 & - & - \\
\hline STR/Biopsy: 1995-2005 (\%) & - & 46 & - & - \\
\hline STR/Biopsy: 2005-2015 $(n=45)$ & - & - & 5 & - \\
\hline STR/Biopsy: 2005-2015 (\%) & - & - & 11 & - \\
\hline Unknown & - & - & - & N/A \\
\hline Unknown: 1985-1995 (n) & 5 & - & - & - \\
\hline Unknown: 1985-1995 (\%) & 1 & - & - & - \\
\hline Unknown: 1995-2005 (n) & - & 2 & - & - \\
\hline Unknown: 1995-2005 (\%) & - & 6 & - & - \\
\hline Unknown: 2005-2015 (n) & - & - & 4 & - \\
\hline Unknown: 2005-2015 (\%) & - & - & 8 & - \\
\hline Chemotherapy $(N)$ & 52 & 35 & 49 & - \\
\hline Received & - & - & - & 0.4397 \\
\hline Received: $1985-1995(n=37)$ & 26 & - & - & - \\
\hline Received: 1985-1995 (\%) & 70 & - & - & - \\
\hline Received: $1995-2005(n=25)$ & - & 21 & - & - \\
\hline Received: 1995-2005 (\%) & - & 84 & - & - \\
\hline Received: $2005-2015(n=49)$ & - & - & 45 & - \\
\hline Received: 2005-2015 (\%) & - & - & 92 & - \\
\hline Not received & - & - & - & 0.563 \\
\hline Not received: $1985-1995(n=37)$ & 11 & - & - & - \\
\hline Not received: 1985-1995 (\%) & 30 & - & - & - \\
\hline Not received: $1995-2005(n=25)$ & - & 4 & - & - \\
\hline Not received: 1995-2005 (\%) & - & 11 & - & - \\
\hline Not received: $2005-2015(n=49)$ & - & - & 4 & \\
\hline Not received: 2005-2015 (\%) & - & - & 8 & \\
\hline Unknown & - & - & - & N/A \\
\hline Unknown: 1985-1995 (n) & 15 & - & - & - \\
\hline
\end{tabular}

Table 3 continues on the next page $\rightarrow$ 
TABLE 3 (continues): Constituents of multidisciplinary management $(N=136)$.

\begin{tabular}{lcccc}
\hline Treatment & 1985-1995 & 1995-2005 & 2005-2015 & $p$-value \\
\hline Unknown: 1985-1995 (\%) & 29 & - & - & - \\
Unknown: 1995-2005 $(n)$ & - & 10 & - & - \\
Unknown: 1995-2005 (\%) & - & 29 & - & - \\
Unknown: 2005-2015 (n) & - & - & 0 & - \\
Unknown: 2005-2015 (\%) & - & - & 0 & - \\
Imagine availability & - & - & - & - \\
CT & Yes & Yes & Yes & - \\
MRI & No & Yes & Yes & - \\
Overall survival & - & - & - & - \\
5-year & - & - & - & $0.0160^{*}$ \\
$\%$ & 51.5 & 49.3 & 76.2 & - \\
10-year & - & - & - & $0.0321^{*}$ \\
$\%$ & 49.5 & 49.1 & 73.2 & - \\
\hline
\end{tabular}

GTR, gross total resection; NTR, near total resection; STR, subtotal resection; 2D, two dimensional; $3 \mathrm{D}$, three-dimensional; $\mathrm{CT}$, computerized tomography; MRI, magnetic resonance imaging.

*, equal or less than $0.05 ; * *$, equal or less than $0.01 ; * * *$, equal or less than 0.001 .

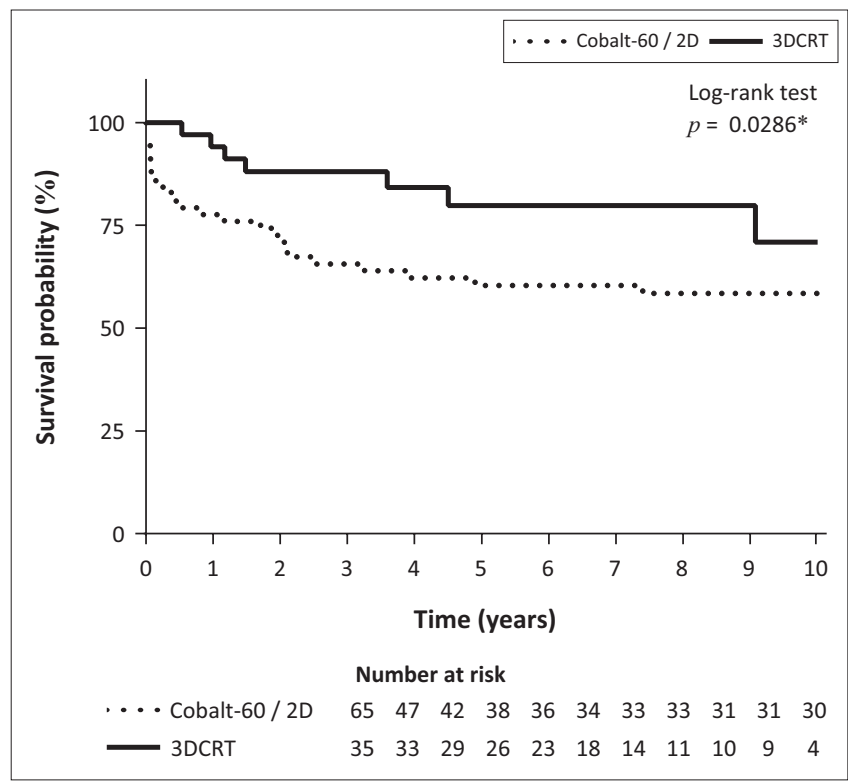

3DCRT, 3D (three-dimnsional) conformal radiotherapy; 2D, two-dimensional.

$*$, equal or less than 0.05 .

FIGURE 3: Comparison of evaluable survival outcomes for those who received 2D radiotherapy on a Cobalt-60 machine (1985-2005) with 3D conformal radiotherapy (3DCRT; 2005-2015) using a linear accelerator $(n=100)$.

technology $(p=0.0216)$, standard-risk MB ( $p=0.0017)$, age 3-15 years $(p=0.0093)$, classic histology $(p=0.0301)$ and GTR $(p=0.0375)$ were all independently associated with hazard ratios that significantly favoured survival. CSF cytology $(p=0.8881)$, sex $(p=0.5985)$ and leptomeningeal spread $(p=0.2935)$ did not significantly influence outcomes.

\section{Discussion}

This single-institution experience describes improvements in the OS of patients with $\mathrm{MB}$, who are treated during the three therapeutic decades, and relates data to advances in the multidisciplinary management of MB in South Africa as representative model of a LMIC. The OS5 and OS10 rates for the entire cohort were $60.5 \%$ and $54.6 \%$, respectively. Survival, without adjusting for risk, was significantly improved in the final decade, with OS5 and OS10 rates of $76.2 \%$ and $73.2 \%$, respectively. The findings of this study are comparable with outcomes achieved in HICs. ${ }^{5,7,12,22}$ Of note is the finding that the relative percentage of standard-risk cases were significantly more during the final decade, highrisk cases remained unchanged and incompletely staged cases declined. This shift in stratification during the final decade may at least in part underpin a survival benefit. The noted frequency of high-risk cases compared with standardrisk cases across the entire cohort was $71.4 \%$, which is much higher than previous reports from HICs where the ratio is almost reversed. ${ }^{17}$ This is most likely the result of various factors that include a large number of patients less than three years old, late presentation because of various socioeconomic and public healthcare factors, delays in definitive diagnosis and multimodal management, and slow advances in diagnostic and treatment approaches. Similar large proportions of high-risk MB cases have been described in other LMICs, including a retrospective synopsis in Morocco, where $70 \%$ of cases were high risk. ${ }^{13}$ Age-adjusted analysis of our data indicated that $59 \%$ of patients under the age group 3-15 years were of high risk, which is in line with other LMIC reports documenting high-risk frequencies of $50 \%-60 \%{ }^{17,23,24}$ Overall, standard-risk MB was significantly less prevalent than the high-risk counterparts and constituted only $28.6 \%$ of our cohort with a OS5 rate of $78.7 \%$, comparable with international outcomes of $80 \%-85 \%$. The OS5 rate of high-risk cases was $51.2 \%$, which is lower than the internationally reported rates of $65 \%-70 \%{ }^{16,17}$

We further quantified survival in the context of the respective risk constituents. The finding that young patients exhibited very poor outcomes was not surprising. Historically, survival rates for this group have been dismal, seldom exceeding $25 \%-45 \%, 8,25,26$ and this is in line with the OS5 rate of $32.5 \%$ we observed. Demographic data suggested that $26.5 \%$ of our cohort were children aged less than three years, which is higher than the reported figures of $10 \%-20 \% .^{27,28}$ Others have suggested that one-third of $\mathrm{MB}$ cases occur before the age of three, which is more consistent with the findings of this study. ${ }^{29}$ The unfavourable outcomes we observed may in part be explained by higher rates of metastatic disease at diagnosis and different underlying biology in young children. Furthermore, the widespread reluctance to expose the immature brain to craniospinal RT and its significant long-term RT effects contribute to removal of sub-optimal tumour. ${ }^{29}$

Metastatic dissemination is characterised by malignant cells in the CSF or leptomeningeal spread on CT or MRI imaging. ${ }^{5,15,26}$ Despite the uncertainty surrounding the origin of CSF sampling in this cohort, the results of this study for combined CSF analysis are consistent with those from other centres using day 14 post-operative lumbar puncture and/or intracranial CSF samples, reporting CSF dissemination in $20 \%-40 \%$ of cases. ${ }^{28,30,31}$ Furthermore, we observed leptomeningeal spread on baseline imaging in $20.0 \%$ of cases, similar to previously reported numbers..$^{26,30,32}$ The findings of this study are in line with previous reports, indicating that the presence of neuraxis dissemination on 


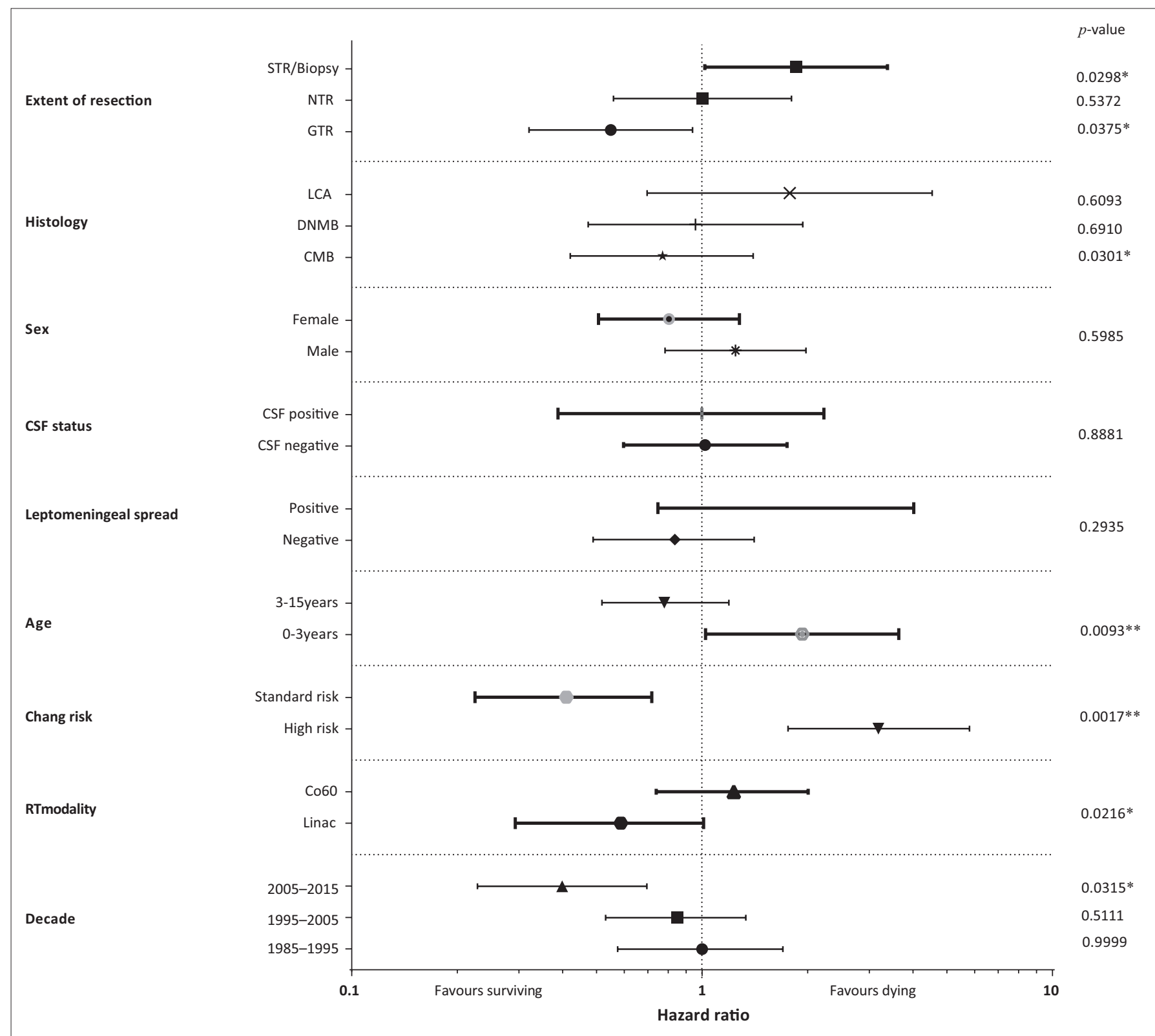

CMB, classic medulloblastoma; DNMB, desmoplastic nodular medulloblastoma; LCA, large cell anaplastic, LM, leptomeningeal; GTR, gross total resection; NTR, near total resection; STR, subtotal resection; CSF, cerebrospinal fluid.

*, equal or less than $0.05 ; * *$, equal or less than 0.01 .

FIGURE 4: Forest plot of univariate analysis for determinants of overall survival. Lines in each row represent $95 \%$ confidence intervals and hazard ratios (HR). The central vertical dotted line indicates the HR for null hypothesis.

MRI correlated with survival, whereas CSF outcomes did not. ${ }^{30}$ The current standard practice includes cytological evaluation of lumbar CSF obtained 14 days post-operatively in conjunction with leptomeningeal assessment on imaging. ${ }^{11}$

In line with previous reports quantifying the proportions of histological variants, the results of this study revealed that classic histology $(72.7 \%)$ was most prevalent, followed by desmoplastic nodular or MBEN (17.2\%) and LCA (10.1\%) histology. This is consistent with other reports, indicating that LCA and desmoplastic nodular or MBEN occur at frequencies of $10 \%-22 \%$ and $7 \%-30 \%$, respectively, with classic tumours constituting the remainder. ${ }^{33}$ Furthermore, LCA histology was associated with inferior OS compared with classic and desmoplastic variants. The OS rates of classic, desmoplastic nodular or MBEN and LCA variants were $66.1 \%, 58.9 \%$ and $39.5 \%$, respectively, which are all lower than the previously reported rates. . $3,17,22^{2}$

Historically, maximal safe resection with GTR, or at least NTR, is considered to be optimal neurosurgical standard of care. Maintaining an appropriate balance between the extent of resection and respect for surrounding organs at risk is critical. This study revealed superior neurosurgical outcomes demonstrated by significantly greater GTR and fewer STR during the third decade of 2005-2015. This may reflect improved surgical techniques over time, or the change in evaluation since in the earlier part of this series, the extent of resection was mostly dependent on the surgical opinion only, or at best contrasted CT assessment. MRI as an early post- 
operative evaluation was only a feature of the later part of the study. The data of this study indicate that GTR was associated with significantly greater OS compared with STR or biopsies, and that the extent of resection does influence survival. Interestingly, Taylor et al. recently reported that the prognostic benefit of the extent of resection may be related to specific molecular subgroups only, and that NTR or even STR might be considered adequate if the likelihood of neurological morbidity is high. ${ }^{9}$ However, in the absence of molecular testing, maximal safe resection remains the standard of care.

It is well established that MB exhibits a male predominance, with a male-to-female ratio of $1.5-1.9 .{ }^{26,34}$ The results of this study, however, revealed a non-significant female predominance with a male-to-female ratio of 0.91 . Although sex is not incorporated into the modified Chang risk classification, it is generally accepted that male outcomes are less favourable. ${ }^{34}$ We observed that male patients had poorer survival outcomes. The data of this study revealed differences in histological variants between male and female patients. Classic histology is associated with more favourable outcomes, and the female cohort of this study consisted of a significantly greater number of this sub-type compared with the male cohort. In contrast, the male sub-cohort consisted of significantly greater LCA variants, which carry the worst prognostic outcome of all the sub-types. Classic histology is known to commonly harbour the WNT molecular subgroup, which is associated with OS greater than $90 \%$, whereas LCA variants commonly harbour the group-3 molecular variant more common in males, which is associated with a high chance of disseminated disease at presentation with an inferior OS of $40 \%-50 \%{ }^{31,35}$ We speculate that these findings may in part explain the poor outcome of male patients in this study, although molecular sub-group identification was not performed in our cohort.

We acknowledge the potential bias of stage migration as previously described by Will Rogers. ${ }^{36}$ This phenomenon may potentially lead to spurious survival statistics. This time-dependent entity may lead to altered staging outcomes and artificial survival increases in both the less and more advanced disease stages. In this study, despite these potential caveats, we show that improved survival was a multifactorial process. During the later phases of this study, diagnostic imaging and pathology were more advanced, a risk-adapted approach was implemented, ${ }^{11}$ CSI was performed using CTbased planning and 3DCRT, chemotherapy was more commonly administered, neurosurgical outcomes improved and accessibility to advanced post-operative imaging was greater. Interpretation of relatively rare disease entities and associated small data sets is limited by the inability to perform accurate multivariate analysis. The alternative is univariate analysis, which we applied to assess the contribution of the various associated risk factors. Univariate analysis may, however, increase the likelihood of type 1 statistical errors and does not allow quantification of each statistical variant's relative contribution to a primary endpoint. Furthermore, the RT modality comparison is confounded as greater numbers of standard-risk diseases, GTR and cases with leptomeningeal spread were observed during the later decade. Improved outcomes are complex and multifactorial.

\section{Conclusion}

We have shown that sequential advances in the multimodal management of MB have benefitted patients in our LMIC setting over the most recent decade, despite a prevalence of high-risk disease. Access to improved imaging modalities, advances in surgical techniques, increased number of patients receiving risk-adapted combination chemotherapy or radiotherapy, as well as CSI using a linear accelerator with 3D planning, are considered as contributing factors. Improved methods to more accurately collect easily accessible clinical data are essential for research, and prospective trials need to be prioritised to address pressing oncological questions. Future work should investigate the effect of reduced-dose CSI on the occurrence of late side-effects and quality of life in the patients of this study. In addition, the ability to perform molecular studies will need to be addressed, as these are providing increasingly powerful evidence for a paradigm shift in the way MBs are approached..$^{37}$

\section{Acknowledgements}

The following people are acknowledged for assistance: Dr Leon Van Wijk (Groote Schuur Hospital) for inspirational discussions, advice and assistance with acquisition of patient records. Dr Omesan Nair (Red Cross Children's Hospital) for assistance with data acquisition. Dr Elvis Kidzeru (Groote Schuur Hospital) for assistance with data collection and verification. Mrs Theo Solomons and Manie Bester (Groote Schuur Hospital) for assistance with acquisition of patient records. Mrs Michelle Kannemeyer (Red Cross Children's Hospital) for assistance with acquisition of patient records. Prof. David Reynders and Mrs Judy Schoeman (Steve Biko Academic Hospital - Paediatric Oncology Unit \& SACCSG registry) for access to medulloblastoma data in the South African Children's Cancer Registry.

\section{Competing interests}

The respective authors report no conflict of interest concerning the research protocols and methods used for this study or the findings outlined in this article.

\section{Authors' contributions}

The following authors contributed to this study: J.R. conducted literature review, data acquisition and statistical analysis, writing, review and editing of the article. A.F. provided neurosurgical support and access or assistance with surgical data. A.F. also assist with the article paper review and edits. A.D. provided paediatric oncology support, guidance, and data. A.D. also performed review and edits of the article. C.S. assisted with data acquisition, analysis, evaluation of first draft, radiation oncology guidance and support. K.P. provided pathology support and evaluation of immunohistochemical outcomes. T.K. provided radiology 
support or expertise, historical feedback, data review and paper assessment. J.P. was the primary supervisor and paediatric radiation oncology support/guidance. J.P. also assisted with Project initiation, access to data or resources, statistical guidance, article reviews and edits.

\section{Funding information}

This research study received no specific grant from any funding agency in the public, commercial or not-for-profit sectors.

\section{Data availability}

No restrictions on data availability. No unique identifiers. Raw data available as Excel spreadsheet on request from the corresponding author.

\section{Disclaimer}

The views and opinions expressed in this research article are those of the authors and do not necessarily reflect the official policy or position of any affiliated agency of the authors.

\section{References}

1. Girardi F, Allemani C, Coleman MP. Worldwide trends in survival from common childhood brain tumors: A systematic review. J Glob Oncol. 2019;5:1-25. https:// doi.org/10.1200/JGO.19.00140

2. Stones DK, De Bruin GP, Esterhuizen TM, Stefan DC. Childhood cancer survival rates in two South African units. S Afr Med J. 2014;104(7):501-504. (In eng) https://doi.org/10.7196/samj.7882

3. Park AK, Lee JY, Cheong $\mathrm{H}$, et al. Subgroup-specific prognostic signaling and metabolic pathways in pediatric medulloblastoma. BMC Cancer. 2019;19(1):571. https://doi.org/10.1186/s12885-019-5742-x

4. Northcott PA, Dubuc AM, Pfister S, Taylor MD. Molecular subgroups of medulloblastoma. Exp Rev Neurother. 2012;12(7):871-884. (In eng). https://doi. org/10.1586/ern.12.66

5. Northcott PA, Korshunov A, Pfister SM, Taylor MD. The clinical implications of medulloblastoma subgroups. Nat Rev Neurol. 2012;8(6):340-351. (In eng). https://doi.org/10.1038/nrneurol.2012.78

6. Northcott PA, Korshunov A, Witt H, et al. Medulloblastoma comprises four distinct molecular variants. J Clin Oncol. 2011;29(11):1408-1414. (In eng). https://doi. org/10.1200/jco.2009.27.4324

7. Louis DN, Perry A, Reifenberger G, et al. The 2016 World Health Organization Classification of Tumors of the Central Nervous System: A summary. Acta Neuropathol. 2016;131(6):803-820. https://doi.org/10.1007/s00401-016-
. Neuropath

8. Ramaswamy $V$, Remke $M$, Bouffet $E$, et al. Risk stratification of childhood medulloblastoma in the molecular era: The current consensus. Acta Neuropathol. 2016;131(6):821-831. (In eng). https://doi.org/10.1007/s00401-016-1569-6

9. Thompson EM, Hielscher T, Bouffet $E$, et al. Prognostic value of medulloblastoma extent of resection after accounting for molecular subgroup: A retrospective integrated clinical and molecular analysis. Lancet Oncol. 2016;17(4):484-495. integrated clinical and molecular analysis. Lance
https://doi.org/10.1016/S1470-2045(15)00581-1

10. Nasir S, Jamila B, Khaleeq S. A retrospective study of primary brain tumors in children under 14 years of age at PIMS, Islamabad. Asian Pacific J Cancer Prev. 2010;11(5):1225-1227. (In eng).

11. Parkes J, Hendricks $M$, Ssenyonga $P$, et al. SIOP PODC adapted treatment recommendations for standard-risk medulloblastoma in low and middle income settings. Pediatr Blood Cancer. 2015;62(4):553-564. (In eng). https://doi.org/ 10.1002/pbc. 25313

12. Katchy KC, Alexander S, Al-Nashmi NM, Al-Ramadan A. Epidemiology of primary brain tumors in childhood and adolescence in Kuwait. SpringerPlus. 2013;2(1):58 (In eng). https://doi.org/10.1186/2193-1801-2-58

13. Elbachiri M, Dao A, Jabir $\mathrm{H}$, et al. [Therapeutic outcomes of medulloblastoma in Casablanca from 2000 to 2012]. Cancer Radiother. 2015;19(8):718-724. (In fre). https://doi.org/10.1016/j.canrad.2015.05.026
14. Ezzat S, Kamal M, El-Khateeb N, et al. Pediatric brain tumors in a low/middle income country: Does it differ from that in developed world? I Neuro-Oncol. 2016;126(2):371-376. (In English). https://doi.org/10.1007/s11060-015-1979-7

15. Harisiadis L, Chang $\mathrm{CH}$. Medulloblastoma in children: A correlation between staging and results of treatment. Int J Radiat Oncol, Biol, Phys. 1977;2(9-10): 833-841. (In eng).

16. Northcott PA, Jones DT, Kool M, et al. Medulloblastomics: The end of the beginning. Nat Rev Cancer. 2012;12(12):818-834. (In eng). https://doi.org/ $10.1038 / \mathrm{nrc3} 410$

17. Gajjar A, Chintagumpala M, Ashley D, et al. Risk-adapted craniospinal radiotherapy followed by high-dose chemotherapy and stem-cell rescue in children with newly diagnosed medulloblastoma (St Jude Medulloblastoma-96): Long-term results from a prospective, multicentre trial. Lancet Oncol. 2006;7(10):813-820. (In eng). https://doi.org/10.1016/s1470-2045(06)70867-1

18. Kruger M, Hendricks M, Davidson A, et al. Childhood cancer in Africa. Pediatr Blood Cancer. 2014;61(4):587-592. (In eng). https://doi.org/10.1002/pbc.24845

19. Muzumdar D, Deshpande A, Kumar R, et al. Medulloblastoma in childhood-King Edward Memorial hospital surgical experience and review: Comparative analysis of the case series of 365 patients. J Pediatr Neurosci. 2011;6(Suppl 1):S78-S85. (In eng). https://doi.org/10.4103/1817-1745.85717

20. Gilbertson RJ, Ellison DW. The origins of medulloblastoma subtypes. Ann Rev Pathol. 2008;3:341-365. (In eng). https://doi.org/10.1146/annurev.pathmechdis. 3.121806.151518

21. Mantel N, Haenszel W. Statistical aspects of the analysis of data from retrospective studies of disease. J Natl Cancer Inst. 1959;22(4):719-748. (In eng).

22. Hoff KV, Hinkes B, Gerber NU, et al. Long-term outcome and clinical prognostic factors in children with medulloblastoma treated in the prospective randomised multicentre trial HIT'91. Eur J Cancer. 2009;45(7):1209-1217. https://doi. org/10.1016/j.ejca.2009.01.015

23. Juraschka K, Taylor MD. Medulloblastoma in the age of molecular subgroups: A review. J Neurosurg Pediatr. 2019;24(4):353-363. https://doi.org/10.3171/2019. 5.PEDS18381

24. Kool M, Korshunov A, Remke M, et al. Molecular subgroups of medulloblastoma: An international meta-analysis of transcriptome, genetic aberrations, and clinical data of WNT, SHH, Group 3, and Group 4 medulloblastomas. Acta Neuropathol. 2012;123(4):473-484. (In eng). https://doi.org/10.1007/s00401012-0958-8

25. Walter AW, Mulhern RK, Gajjar A, et al. Survival and neurodevelopmental outcome of young children with medulloblastoma at St Jude Children's Research Hospital. J Clin Oncol. 1999;17(12):3720-3728. (In eng). https://doi.org/10.1200/ JCO.1999.17.12.3720

26. Millard NE, De Braganca KC. Medulloblastoma. J Child Neurol. 2015;31(12): 1341-1351. (In Eng). https://doi.org/10.1177/0883073815600866

27. Ramaswamy $V$, Remke $M$, Bouffet $E$, et al. Recurrence patterns across medulloblastoma subgroups: An integrated clinical and molecular analysis.
Lancet Oncol. 2013;14(12):1200-1207. (In eng). https://doi.org/10.1016/s1470Lancet Oncol. 2013:13)70449-2

28. Lee DS, Cho J, Kim SH, et al. Patterns of failure following multimodal treatment for medulloblastoma: Long-term follow-up results at a single institution. Cancer Res medulloblastoma: Long-term follow-up results at a single institution. Can
Treat. 2015;47(4):879-888. (In eng). https://doi.org/10.4143/crt.2014.067

29. Von Bueren AO, Von Hoff K, Pietsch T, et al. Treatment of young children with localized medulloblastoma by chemotherapy alone: Results of the prospective, multicenter trial HIT 2000 confirming the prognostic impact of histology. Neurooncology. 2011;13(6):669-679. (In eng). https://doi.org/10.1093/neuonc/nor025

30. Terterov S, Krieger MD, Bowen I, McComb JG. Evaluation of intracrania cerebrospinal fluid cytology in staging pediatric medulloblastomas, supratentorial primitive neuroectodermal tumors, and ependymomas. J Neurosurg Pediatr. 2010;6(2):131-136. (In eng). https://doi.org/10.3171/2010.5. peds09333

31. Taylor MD, Northcott PA, Korshunov A, et al. Molecular subgroups of medulloblastoma: The current consensus. Acta Neuropathol. 2012;123(4): 465-472. (In eng). https://doi.org/10.1007/s00401-011-0922-z

32. Gajjar A, Fouladi M, Walter AW, et al. Comparison of lumbar and shun cerebrospinal fluid specimens for cytologic detection of leptomeningeal disease in pediatric patients with brain tumors. J Clin Oncol. 1999;17(6):1825-1828. (In eng). https://doi.org/10.1200/JCO.1999.17.6.1825

33. Gulino A, Arcella A, Giangaspero F. Pathological and molecular heterogeneity of medulloblastoma. Curr Opin Oncol. 2008;20(6):668-675. https://doi.org/ 10.1097/CCO.0b013e32831369f4

34. Weil MD, Lamborn K, Edwards MS, Wara WM. Influence of a child's sex on medulloblastoma outcome. JAMA. 1998;279(18):1474-1476. (In eng). https:// doi.org/10.1001/jama.279.18.1474

35. Schroeder K, Gururangan S. Molecular variants and mutations in medulloblastoma. Pharmacogen Pers Med. 2014;2014(7):43-51. (In eng). https://doi.org/10.2147/ pgpm.s38698

36. Feinstein AR, Sosin DM, Wells CK. The Will Rogers phenomenon. Stage migration and new diagnostic techniques as a source of misleading statistics for survival in cancer. N Engl J Med. 1985;312(25):1604-1608. https://doi.org/10.1056/ NEJM198506203122504

37. Rajeshwari M, Kakkar A, Nalwa A, et al. WNT-activated medulloblastoma with melanotic and myogenic differentiation: Report of a rare case. Neuropathol. 2016;36(4):372-375. https://doi.org/10.1111/neup.12281 


\section{Appendix 1}

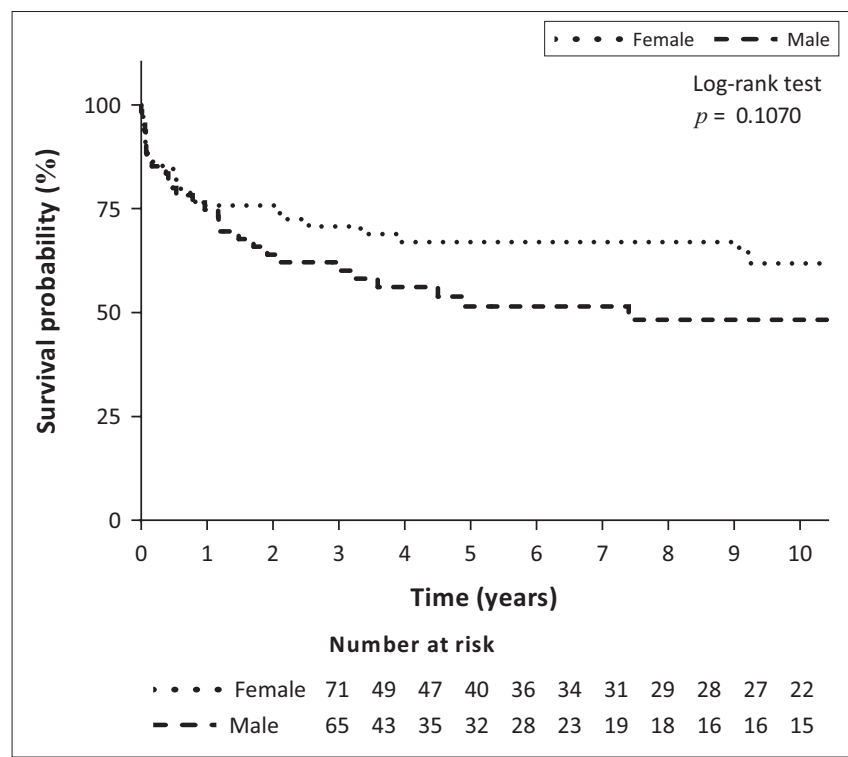

FIGURE 1-A1: Ten-year overall survival comparison between female and male patients $(n=136)$.
TABLE 1-A1: Comparison of risk factors between female and male patients. The evaluable size of each sub-cohort is indicated $(n)$.

\begin{tabular}{|c|c|c|c|c|c|}
\hline \multirow[t]{2}{*}{ Variable } & \multicolumn{2}{|c|}{ Female } & \multicolumn{2}{|c|}{ Male } & \multirow{2}{*}{$\begin{array}{c}p \text {-value } \\
\text { (Chi-square } \\
\text { test) }\end{array}$} \\
\hline & $n$ & $\%$ & $n$ & $\%$ & \\
\hline Number $(n=136)$ & 71 & 52.2 & 65 & 47.8 & 0.3960 \\
\hline Median age at diagnosis (years) & 5.5 & - & 6.0 & - & - \\
\hline Number deceased (confirmed) & $23 / 71$ & 32.4 & $31 / 65$ & 47.7 & 0.0807 \\
\hline \multicolumn{6}{|l|}{ Histology $(n=128)$} \\
\hline Classic & $55 / 67$ & 82.1 & $38 / 61$ & 62.3 & $0.0168^{*}$ \\
\hline Desmoplastic & $10 / 67$ & 14.9 & $12 / 61$ & 19.7 & 0.4928 \\
\hline LCA & $2 / 67$ & 3.0 & $11 / 61$ & 18.0 & $0.0068 * *$ \\
\hline \multicolumn{6}{|l|}{ Extent of resection ( $n=125$ ) } \\
\hline GTR & $24 / 67$ & 34.3 & $25 / 58$ & 41.7 & 0.1562 \\
\hline NTR & $23 / 67$ & 32.9 & $19 / 58$ & 31.7 & 0.3291 \\
\hline STR & $18 / 67$ & 25.7 & $14 / 58$ & 23.3 & 0.2985 \\
\hline Biopsy & $5 / 67$ & 7.1 & $2 / 58$ & 3.3 & 0.1973 \\
\hline CSF dissemination $(n=93)$ & $10 / 51$ & 19.6 & $12 / 42$ & 28.6 & 0.3369 \\
\hline Leptomeningeal spread $(n=90)$ & $12 / 50$ & 24 & $6 / 40$ & 15.0 & 0.1923 \\
\hline Chemotherapy received $(n=136)$ & - & 86.9 & - & 80.1 & 0.4892 \\
\hline Radiotherapy received $(n=100)$ & - & 77.7 & - & 79.8 & 0.3091 \\
\hline High-risk disease $(n=117)$ & - & 68.3 & - & 73.6 & 0.5472 \\
\hline
\end{tabular}

GTR, gross total resection; NTR, near total resection; STR, subtotal resection; CSF, cerebrospinal fluid; LCA, large cell anaplastic.

$*$, equal or less than $0.05 ; * *$, equal or less than 0.01 . 